\title{
High-Resolution Phase Based Method for FMCW Short Range Radars
}

\author{
Mikhail V. Ronkin ${ }^{1, \text { a) }}$, Alexey A. Kalmykov ${ }^{1}$, Elena N. Akimova ${ }^{1,2}$, \\ Victor S. Nagovicin ${ }^{3}$ \\ ${ }^{1}$ Yeltsin Ural Federal University, Yekaterinburg, Russia \\ ${ }^{2}$ N. N. Krasovskii Institute of Mathematics and Mechanics, Ural Branch of RAS, Ekaterinburg, Russia \\ ${ }^{3}$ Ural State University of Railway Transport, Ekaterinburg, Russia \\ ${ }^{\text {a)} C o r r e s p o n d i n g ~ a u t h o r: ~ m v R o n k i n @ g m a i l . c o m ~}$
}

\begin{abstract}
The paper deals with the problem of the using phase-based methods for frequency estimation in the frequency modulated continuum waves (FMCW) short-range radars in the case of high-resolution frequency estimation requirements. The equation is considered for frequency estimation by the least-square method of approximating the phase-to-time relation of the beat signal correlation function in the case of several significant components in the signal (such as valuable and interference signal-like tones). Solution is proposed for the equations by using the parametric (or subspace) decomposition of beat signals (such as eigenvector decomposition, EV). The numerical investigation shows that the bias of the frequency estimations by the proposed solution of the mentioned equation above has statistical properties similar to the method of estimation of signal parameters via rotational invariance techniques (ESPRIT). However, the proposed method does not require the double decompositions, and frequency of each eigenvector can be estimated separately. It is also shown that in the case of the unknown number of signal components the proposed method has the higher statistical properties than for such popular technique as the multiple signal classification method (MUSIC).
\end{abstract}

Keywords: FMCW signals, short range radars, FMCW radars, signal processing, frequency estimation, high-resolution frequency estimation, time delay measurements.

\section{INTRODUCTION}

One of the main problems of using the short-range radars in industry is increasing of the resolution of valuable signals on the background of close interference signals. For instance, such a problem has a place in the radar level measurements of small tank filling level, when the bottom of the tank is the parasitic reflector [1]. The other examples when the discussed problem appears are: ultrasonic time-of-flight flow measurements with clamp-on transducers in the case of poorly matched media [2], radar vitality monitoring [3], and many other cases.

As a rule, in the mentioned above and other short-range radar applications, the complex modulated signals such as linear frequency modulated continuum waves (FMCW) are applied. Such signals allow one to reach signal-tonoise ratio higher than for the pulse ones with the same power of emitting. Frequently in short-range FMCW radars signals are processed by so-called heterodyne scheme of a transmitter. The principle of the heterodyne scheme is shown in the Fig.1. In the scheme in Fig. 1, the emitted (reference) and received FMCW signals $\left(s_{\mathrm{fmcw}}\right)$ are multiplied by the heterodyne with followed low- or band- pass filtration of the low-frequency part. The resulted signal is the so-called beat signal ( $s_{\text {beat }}$ ), which contains the information about the signal time delay in the frequency and initial phase. In the general case, quadrature heterodyne processing can be performed. In this case, both the real and imaginary part of the beat signal would be obtained separately [1].

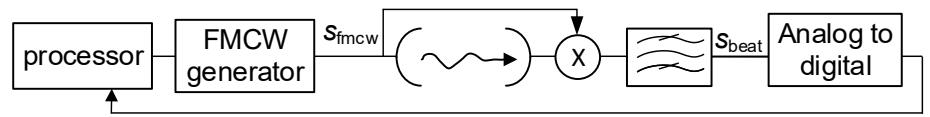

FIGURE 1. Block-scheme of the heterodyne based transceiver

International Conference of Numerical Analysis and Applied Mathematics ICNAAM 2019 AIP Conf. Proc. 2293, 140003-1-140003-4; https://doi.org/10.1063/5.0026619 Published by AIP Publishing. 978-0-7354-4025-8/\$30.00 
The analytical model of the beat signal has the following expression

$$
s_{b}(t)=A_{b} \exp \left\{i\left(2 \pi f_{b} t+\theta_{b}\right)\right\}=A_{b} \exp \left\{i\left(2 \pi\left(\Delta f \tau / T_{m}\right) t+2 \pi f_{0} \tau\right)\right\} .
$$

where $A_{b}$ is the amplitude of the beat signal; $f_{b}, \theta_{b}$ frequency and initial phase of the beat signal; $f_{0}$ is the initial frequency; $\Delta f$ is the frequency deviation; $T_{m}$ is the period of modulation.

As a rule, in signal (1) only the beat frequency is measured. In this case, the task of increasing the signals resolution can be reduced to the task of increasing frequency (or spectral) resolution. There are many approaches have been proposed for this task solution in the literature [4,5]. Analysis of the literature shows that one of the most popular approaches for increasing the frequency resolution is based on the signal subspace decomposition techniques like the eigenvector decomposition (EV) [1].

The EV based methods allow one to obtain the so-called super-resolution of the signal components. However, such methods have high computational complexity. For instance, EV has computational complexity of order $O\left(N^{2} M\right)$, where $\mathrm{N}$ is the digitized signal sample length, $\mathrm{M}$ is the number of eigenvectors under consideration. Therefor such popular techniques as Multiple Signal Classifier method (MUSIC) and Estimation of Signal Parameters via Rotational Invariance Technique (ESPRIT) have computational complexity order at least $O\left(2 N^{3}\right)$, if $M=N$ [6]. Another problem of this group of methods is the task of choosing the order of the model. The incorrect order model could lead to rising of the errors of frequency estimation. In particular this problem actual in the MUSIC based methods. Analysis of the problem of high-resolution delay estimation in the FMCW radars shows the actuality of investigation of new methods for the parametric spectral resolution, which have to be robust to the incorrect model order and minimize computational complexity of their implementation.

\section{PROBLEM FORMULATION}

As it was shown in works $[2,7]$ the beat signal in the short range FMCW radar systems could be written as amplitude-modulated superposition of narrowband valuable and interference signal-like terms on the background of the white Gaussian noises. As a rule, such systems provide sufficiently high signal-to-noise ratio and the most part of interferences could be filtered. However, as it was shown above in some application the case when high resolution is required is of the high interest. The beat signal of the short range FMCW radar can be described as

$$
s(n)=\sum_{p=1}^{P} A_{p} \exp \left\{\mathrm{j}\left(\omega_{p} n+\theta_{p}\right)\right\}+z(n),
$$

where $p=1, \ldots, P$ is the number of terms; $A_{p}$ is the amplitude of the $p$-th term (with amplitude modulation); $\mathrm{z}(\mathrm{n})$ is the Gaussian white noise (additive interferences); $\omega_{p}, \theta_{p}$ are the frequency and phase of $\mathrm{p}$-th component; $\omega_{p}=2 \pi f_{b p} / f_{s}$, where $f_{b p}-$ beat frequency of the $p$-th component; $f_{s}-$ sampling frequency; $n=0, \ldots, N-1-$ samples of the discrete time.

Using the approach proposed in $[2,7,8,9]$ distribution of estimation results $\left\{\widehat{\omega}_{p}\right\}_{p=\overline{1, P}}$ by autocorrelation function of beat signal has the following form:

$$
P(n) \sim\left(1 / \sigma_{0}^{2}\right) \exp \left\{\sum_{n=1}^{N}\left[R-\sum_{p=1}^{P} e^{j \widehat{\omega}_{p} n}\right]^{2}\right\},
$$

where $P(n)$ is the probability distribution of $\omega_{p}$ estimation; $\sigma_{0}^{2}$ is the variance of noises $z(n) ; R$ is the autocorrelation function of (2); $\widehat{\omega}_{p}$ is the estimation of frequency of $p-$ th component.

As it was shown in [9] in the case when $p=1$, the expression (3) corresponds to the estimation of the signal parameter by peak searching of the Fourier spectrum. In the general case, expression (2) could be written as $[2,8]$ :

$$
\mathrm{R}(\mathrm{n})=\sum_{\mathrm{p}=1}^{\mathrm{P}}\left|\mathrm{R}_{\mathrm{p}}(\mathrm{n})\right| \exp \left\{\mathrm{j} \omega_{\mathrm{p}} \mathrm{n}\right\}+\mathrm{z}(\mathrm{n}) \text {. }
$$

In expression (4) $\left|R_{p}(n)\right| \gg z(n)$, as it shown in [2, 10] the expression (4) transformed to:

$$
\mathrm{R}(\mathrm{n})=\sum_{\mathrm{p}=1}^{\mathrm{P}}\left|\mathrm{R}_{\mathrm{p}}(\mathrm{n})\right| \exp \left\{\mathrm{j} \omega_{\mathrm{p}} \mathrm{n}+\mathrm{j} \mathrm{I}_{\mathrm{I}}(\mathrm{n})\right\}=\sum_{\mathrm{p}=1}^{\mathrm{P}}\left|\mathrm{R}_{\mathrm{p}}(\mathrm{n})\right| \exp \left\{\mathrm{j} \arg \mathrm{R}_{\mathrm{p}}(\mathrm{n})\right\}
$$

where $v_{I p}(n)$ is the noise component (phase noises) which corresponds to the $\mathrm{z}(\mathrm{n})$ influence on the signal; $\arg R_{p}(n)=\omega_{\mathrm{p}} n+v_{I_{p}}(n)$ is the phase of autocorrelation function for $p-t h$ signal component.

Zeros of the derivative of (3) correspond to the maximum likelihood estimations for $\omega_{\mathrm{p}}$. By using (5) in the logarithm of (3) the derivative of $P(n)$ can be written as follows (see [2, 10]):

$$
\partial|\log (P(n))| / \partial \omega_{p} \sim \operatorname{Im}\left\{\left(2 / \sigma_{0}^{2}\right) \sum_{n=1}^{N} \sum_{p=1}^{P}\left|R_{p}\right| n \exp \left[j\left(\arg R_{p}-\widehat{\omega}_{p} n\right)\right]\right\}=0 .
$$

By using the Taylor decomposition of $e^{x} \approx 1+x$ near the value $\widehat{\omega}_{p} \approx \omega_{p}$ expression (6) leads to the follows equation $($ see $[2,10])$ :

$$
\sum_{n=1}^{N} \sum_{p=1}^{P}\left|R_{p}\right| n^{2} \widehat{\omega}_{p}=\sum_{n=1}^{N} \sum_{p=1}^{P}\left|R_{p}\right| n \arg R_{p}
$$


Expression (7) corresponds to the equation, which solution allows obtaining $\widehat{\omega}_{p}$ by the least square method $[2,10]$. In the case of $p=1$ or when the amplitude of valuable component (note it $\left|\mathrm{R}_{0}\right|$ ) much greater than for interference ones $\left(\left|R_{0}\right|>\left|R_{p}\right|, \forall p \neq 0\right)$ the solution of (7) provides the weighted least square estimator of $\widehat{\omega}_{0}$, which was obtained in [7]

$$
\widehat{\omega}_{0}=\sum_{\mathrm{n}=0}^{\mathrm{N}-1} \mathrm{n}\left|\mathrm{R}_{0}(\mathrm{n})\right| \arg \left\{\mathrm{R}_{0}(\mathrm{n})\right\} / \sum_{\mathrm{n}=0}^{\mathrm{N}-1} \mathrm{n}^{2}\left|\mathrm{R}_{0}(\mathrm{n})\right| .
$$

In the general case, equation (7) does not have an analytical solution. If it is necessary to take more than one component in (7), its solution could be found by applying some decomposition technique and using (8) for each obtained term. Such decomposition could be performed by the EV or EMD methods.

\section{NUMERICAL INVESTIGATION}

The numerical experiments had the aim to investigate the bias of the frequency estimation for results of the parametric decomposition of beat signals under the typical conditions for the short-range industrial radar systems. The decomposition has been performed by the EV and EMD methods for proposed estimator (8) ( prop $_{\mathrm{EV}}$ for EV and $\operatorname{prop}_{\mathrm{EMD}}$ for the EMD decomposition). Also, the estimator (8) using the hypothesis that $\left|\mathrm{R}_{0}(\mathrm{n})\right|=$ const $\left(F i t z_{\mathrm{EV}}\right)$ was investigate, such estimator corresponds to the well-known Fitz estimator [8] $\widehat{\omega}_{0}=\sum_{\mathrm{n}=0}^{\mathrm{N}-1} \mathrm{n} \arg \left\{\mathrm{R}_{0}(\mathrm{n})\right\} / \sum_{\mathrm{n}=0}^{\mathrm{N}-1} \mathrm{n}^{2}$. The analyzed estimators has also been compared with estimation of (8) (prop $\left.p_{\mathrm{f}}\right)$ without decomposition and with results obtained by root-MUSIC (MUSIC) and ESPRIT (ESPRIT) methods [11]. In all experiments, the beat signal was simulated as the amplitude-modulated superposition of valuable signal with amplitude 1 and frequency from 0 to 1 in relative units and signal-like interferences with amplitudes 0.99 and frequencies about 1 in relative units. All signals have classical resolution 0.01 in relative units. Amplitude modulation was set as $40 \%$.

Figures 2 and 3 show the relations of the bias of valuable frequency estimation to its frequency value in the range $0-1(\delta, \%)$ obtained by analyzed methods. Fig. 2 a,b shows relations for one interference signal with frequency 1 for chosen order of the model $\mathrm{p}=1$ (Fig. 2 a) and $\mathrm{p}=1$ (Fig. 2 b). The Fig. 3 a-c shows relations for two interference signals with frequencies 1 and 1.09 and same amplitude 0.99 for chosen order of the model p=1 (Fig. 3a), p=2 (Fig. $3 b)$ and $p=3$ (Fig. 3c).

Analysis of the obtained results (see Fig. 2,3) shows the advantages of the proposed estimator prop $_{\mathrm{EV}}$ in the case of unknown number of signal components. The additional advantages of $\operatorname{prop}_{\mathrm{EV}}$ that it is not necessary to use the full decomposition of the signal because the frequency of each component can be estimated separately. It should be noted that in the case of the correct order of the model estimation by MUSIC methods allows reaching more accurate results than for other investigated ones(see Fig. $2 \mathrm{~b}$ and $3 \mathrm{c}$ ), however this case very rare in the practice. It is also should be noted that statistical behavior of ESPRIT estimator is similar to the proposed ones ( rrop $_{\mathrm{EV}}$ ) in the case of the correct order of the model (see Figs. 2 a and $3 \mathrm{a}, \mathrm{b}$ ). The results without decomposition ( prop $_{\mathrm{f}}$ ) are the same as for, EMD ( $\operatorname{rrop}_{\mathrm{EMD}}$ ). The results for estimators Fit $_{\mathrm{EV}}$ and $\operatorname{prop}_{\mathrm{EV}}$ are differ less than 0.1, however, in the general case prop $_{\mathrm{EV}}$ could be recommended as main method due to the similar computational complexity of both methods.

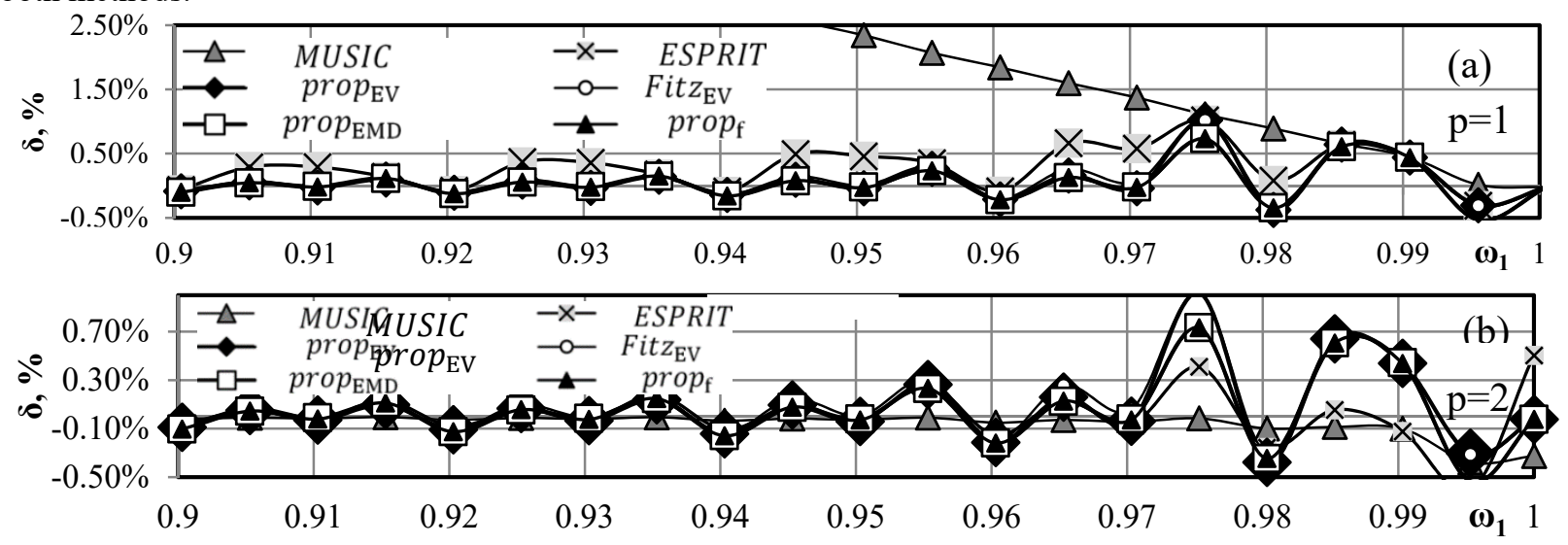

FIGURE 2. Relations of bias $(\delta, \%)$ of valuable frequency estimation to its frequency $(\omega 1)$ value in the range $0-1$ obtained by the analyzed methods for the one interferernce signal and for the model order $p=1$ (a) and $p=2$ (b) 


\section{CONCLUSION}

The Implemented investigation of the high-resolution phase-based method for FMCW short-range radars shows the possibility of using the proposed phase-based method for the parametric (or sub-space) decomposition of beat signals. The numerical results show the advantages of the proposed approach in the case of an unknown order of parametric model of the high-resolution frequency estimation. It is shown, that the statistical behavior of the proposed estimator similar to the ESPRIT method. However, the proposed method has less computational complexity and does not require correct choice of the order of the model.
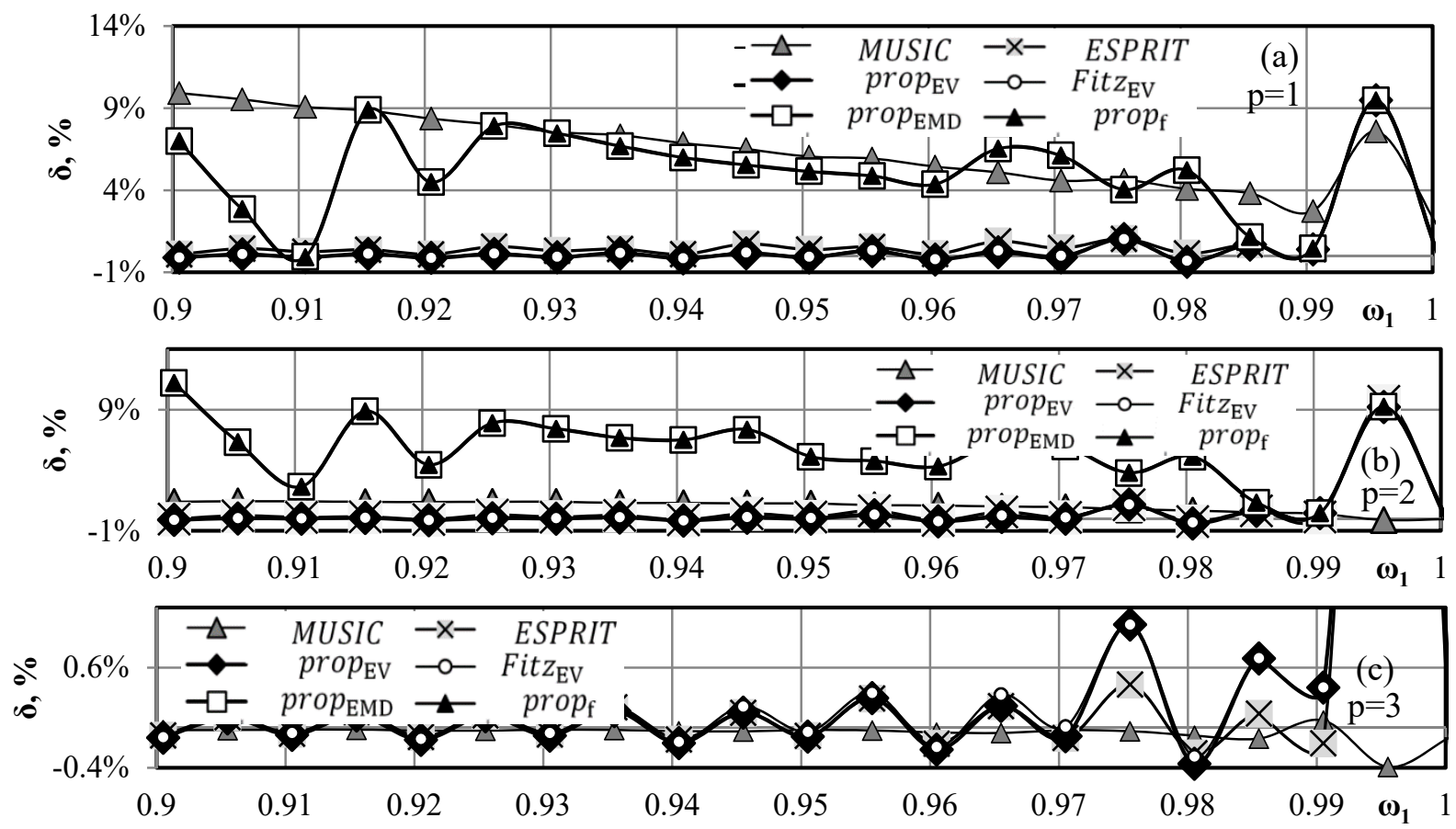

FIGURE 3. Relations of bias $(\delta, \%)$ of valuahle frequency estimation to its frequency $(\omega 1)$ value in the range $0-1$, obtained by the analyzed methods for two interference signals and for the model of order $p=1$ (a), $p=2$ (b) and $p=3$ (c)

\section{REFERENCES}

1. B.A. Atayants, V.M. Davydochkin, V.V. Ezerskiy, et al, Precision systems of FMCW short-range radar for industrial applications. ARTECH HOUSE USA (2014).

2. M.V. Ronkin, A.A. Kalmykov, "Investigation of the time delay difference estimator for FMCW signals", Proceedings of the 2nd International Workshop on Radio Electronics \& Information Technologies (REIT 2 2017), 90-99 (2017) http://ceur-ws.org/Vol-200.

3. M.C. Shastry, "Algorithms for implementing Hilbert Huang transform for through wall human activity detection", http://geogin.narod.ru/hht/link09/read9-7.pdf.

4. P.Stoica, "List of references on spectral line analysis", Signal Processing, vol. 31 (3), 329-340 (1993).

5. F.Castanie, Digital Spectral analysis. parametric, non-parametric and advanced methods. ISTE Ltd, (2011) .

6. C. Stockle, J. Munir, A. Mezghani, J.A. Nossek, "DoA Estimation Performance and Computational Complexity of Subspace- and Compressed Sensing-based Methods", 19th Inter. ITG Workshop on Smart Antennas (WSA 2015), (2015).

7. M.V. Ronkin, A.A. Kalmykov, "Phase based frequency estimator for short range FMCW radar systems", 2018 Ural Symposium on Biomedical Engineering, Radioelectronics and Information Technology (USBEREIT), 367-370 (2018).

8. D. Rife and R. Boorstyn, "Single-tone parameter estimation from discrete-time observations", IEEE Trans. on Information Theory, vol. 20 (5), 591-598 (1974).

9. M.P. Fitz, "Further Results in the Fast Estimation of a Single Frequency", IEEE trans. on communications, vol 42, 862-864 (1994).

10. S. A. Tretter, "Estimating the frequency of a noisy sinusoid by linear regression", IEEE Trans. Inform. Theory, vol IT-3 1, 832-835 (1985).

11. P.Stoica, R.Moses, Spectral analysis of signal. PRENTICE HALL (2005). 